\title{
A New Version of the Plasma Database for Plasma Physics in the Data Center for Plasma Properties
}

\author{
Received January 24, 2020; accepted January 31, 2020
} Jun-Hyoung Park*, Heechol Choi, Won-Seok Chang, Sang Young Chung, Deuk-Chul Kwon, Mi-Young Song, and
Jung-Sik Yoon

Plasma Technology Research Center, National Fusion Research Institute, Gunsan 54004, Republic of Korea

*Corresponding author E-mail: pjh1126@nfri.re.kr

\begin{abstract}
Bulk and surface chemistry database (DB) are necessary to compute plasma parameters using plasma simulators. As the high quality of the DB is closely related to the accuracy enhancement of simulations, we attempted to gather reliable data from previously published articles. However, previous systems could not accommodate various types of information such as electron collision cross sections, rate coefficients of heavy particle reactions, sticking coefficients on the surfaces, and thermodynamic data. Therefore, we developed a new version of the DB system to provide plasma data for researchers in related fields; this new DB system is designed to retrieve data such as electron collision cross sections, rate coefficient, and thermodynamics data. About 35,000 collision cross sections and 74,000 rate coefficients for 90 chemical species and thermodynamic data for 55 chemicals are provided in the numerical and graphical forms in this DB system.
\end{abstract}

Keywords: Atomic and molecular data, Plasma database, Plasma chemistry, Plasma properties

\section{Introduction}

Recently, the importance of the plasma and plasma-material interaction data is growing because of the need to understand plasma characteristics in the field of nuclear fusion and process plasmas [1]. Bulk and surface chemistry databases (DBs) have become necessary to compute plasma parameters using plasma simulators. The high quality of a DB is closely related with the accuracy enhancement of the simulations, and therefore, considerable research has been invested in attempting to design a reliable $\mathrm{DB}$.

The International Atomic Energy Agency (IAEA) supports the atomic and molecular data (AMD) unit within its nuclear data section, wherein the primary objective is to establish and maintain internationally recommended numerical DBs on atomic and molecular collision and radiative processes; atomic and molecular structure characteristics; particle-solid surface interaction processes; physical, chemical, and thermo-mechanical material properties for use in fusion energy research; and other plasma science and technology applications [2]. Currently, the AMD unit operates two DB systems: ALADDIN and AMBDAS. The former provides numerical data for collision and particle-surface interaction data, and the latter furnishes bibliographic information on atomic, molecular, and plasma-material interactions data, and surface reactions. Most of the data have been collected or generated through internal or external professional consultant meetings. Further, the AMD unit operates a search engine called GENIE with which data on cross sections and rate coefficients are searched. The engine can search such data using several internal and external related databases such as IAEA ALADDIN, NIFS AMDIS, CAMBD, NIST atomic cross sections, OPEN-ADAS, and Spectr-W3. However, as mentioned above, the AMD unit provides nuclear data, and the DBs are not constantly updated [3-8].

VAMDC connects over 30 related DB systems to provide atomic molecular DBs. Most of these DBs deal with data used in astronomy spectral analysis and modeling in many fields of astrophysics. In addition, the e-infrastructure of VAMDC for data sharing has been updated since 2011. The basic data sharing form uses an extensible markup language (XML) template called XSAMS, which provides a standardized approach to atomic molecular information. The DBs related to atomic and molecule data connected to VAMDC are ALADDIN and LXCat [9-12].

Quantemol (QDB) is a plasma process DB developed by the University College London in 2016, and it contains chemical data for plasma chemistry modeling. The QDB aims to provide an effective environment for collaborative research between schools and industry in the field of plasma modeling [13].

The LXCat project aims to provide a web-based platform to collect data required for low-temperature plasma modeling. The main data provided are electron scattering cross sections, differential scattering cross sections, rate coefficients, and ion scattering cross sections. In addition, each contributor is responsible for the data problem, as this $\mathrm{DB}$ is maintained by the data submitted by contributors [14].

The data center for plasma properties (DCPP) of the National Fusion Research Institute (NFRI, Korea) has been collecting and disseminating experimental and research data related to plasma properties since 2006. We have accumulated about 100,000 plasma properties data [15]. Further, to improve the reliability of data, objective evaluations have been conducted by experts. Some of these data have been certified as standard reference data from the National Center Standard 
Reference Data (NCSRD) of the Korean Agency for Technology and Standards, and these data have been registered in the National Standard Reference DB [16]. In addition, for the efficiency of data acquisition, ISO9001: 2015 certification has been obtained and applied to detailed work processes. However, the previous DB system could not accommodate various types of information on electron collision cross sections, rate coefficients of heavy particle reactions, sticking coefficients on the surfaces, and thermodynamic data, and therefore, we developed a new version of the DB system to provide plasma data for researchers from related fields. In this work, we briefly introduce the DB system for providing plasma properties data to plasma researchers.

\section{Database (DB) system and discussion}

\subsection{Overview}

Figure 1 shows a search page of the previous system. Users could select and search for data based on the target particle and impact method. Because the previous system supported electron collision cross section, the system only required defining the target and projectile particles. Here, projectile particle was "e" or "hv." However, it was difficult to obtain the desired data because a considerable amount of data was retrieved. Therefore, reordering or reselection of data was required to select a specific process from the searched list.

We developed a new system for plasma properties to solve the problems of the previous system. As shown in Fig. 2, the proposed system is easy to use and find the desired data. Moreover, the new

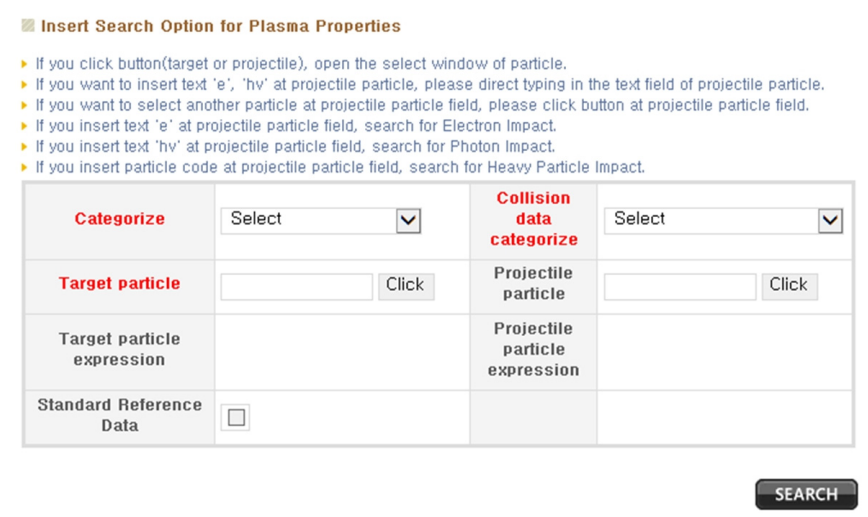

Figure 1. (Color online) Search page of previous system.

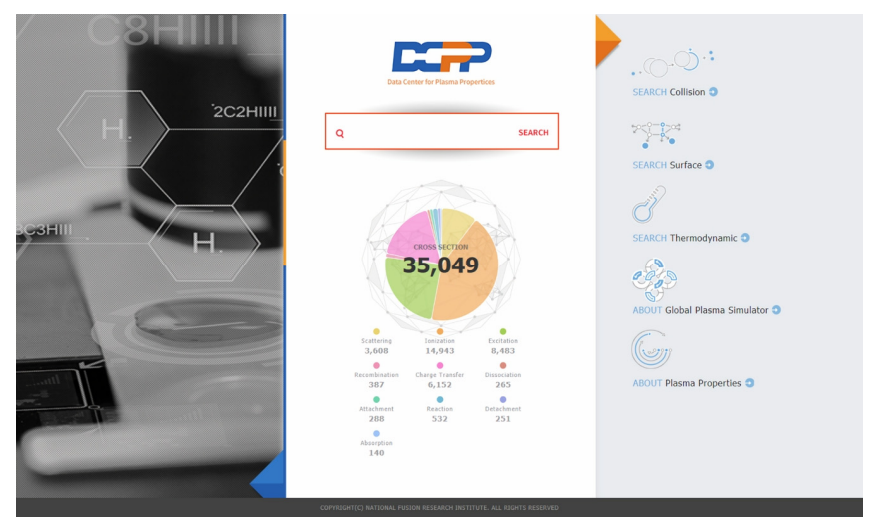

Figure 2. (Color online) Main page of the new system. system can be easily accessed from various devices because it supports HTML5.

Most data have been obtained from previously published articles. In addition, evaluated data provided by experts were uploaded according to the certification data evaluation process of Korea NCSRD. Thermodynamic data were calculated using quantum chemistry methods with density functional theory (DFT), Post-HF, EOM-IP, EOM-EA etc. implemented in quantum chemistry calculation programs such as Gaussian09, GAMESS, Cfour, MolPro, and KisTheIP. Therefore, the ground state structure, electron affinity, ionization potential, polarizability, Lennard-Jones parameter, thermodynamic data, chemical reaction path, energetic properties, excited electronic states geometries, and energy characteristic data were presented through the search.

\subsection{Searchable data}

Table I shows the statics of collision cross sections and rate coefficients in the DB. Thus far, about 100,000 data of collision cross section and rate coefficient were accumulated in the DB. A total of 90 species were evaluated by experts as summarized in Table II, and the number of evaluated DBs is $\sim 700$. Tables III and IV summarize the available lists of surface reaction data and thermodynamic data, respectively. Surface reaction data is collected from papers published in journals. As mentioned above, thermodynamic data were obtained using quantum chemistry methods.

Table I. Statistics of collision process data.

\begin{tabular}{|c|c|c|c|c|}
\hline & Type & & & \\
\hline \multirow{22}{*}{$\begin{array}{l}\text { Collision } \\
\text { Processes }\end{array}$} & \multirow{10}{*}{ Cross section } & Absorption & 140 & \multirow{10}{*}{34799} \\
\hline & & Attachment & 288 & \\
\hline & & Charge transfer & 5892 & \\
\hline & & Detachment & 251 & \\
\hline & & Dissociation & 265 & \\
\hline & & Excitation & 8483 & \\
\hline & & Ionization & 14934 & \\
\hline & & Reaction & 532 & \\
\hline & & Recombination & 387 & \\
\hline & & Scattering & 3610 & \\
\hline & \multirow{2}{*}{$\begin{array}{l}\text { Differential } \\
\text { cross section }\end{array}$} & Excitation & 2 & \multirow{2}{*}{4} \\
\hline & & Ionization & 2 & \\
\hline & \multirow{10}{*}{ Rate coefficient } & Absorption & 5 & \multirow{10}{*}{69210} \\
\hline & & Attachment & 446 & \\
\hline & & Charge transfer & 1314 & \\
\hline & & Detachment & 10 & \\
\hline & & Dissociation & 230 & \\
\hline & & Excitation & 29580 & \\
\hline & & Ionization & 1255 & \\
\hline & & Reaction & 3729 & \\
\hline & & Recombination & 32511 & \\
\hline & & Scattering & 130 & \\
\hline
\end{tabular}


Table II. Statistics of evaluated species and process.

\begin{tabular}{|c|c|c|c|c|c|c|c|c|c|c|}
\hline Group & Atoms & $\begin{array}{l}\text { Diatomic } \\
\text { molecules }\end{array}$ & $\begin{array}{l}\text { Triatomic } \\
\text { molecules }\end{array}$ & $\begin{array}{l}\text { Tetratomic } \\
\text { molecules }\end{array}$ & $\begin{array}{c}\text { Pentatomic } \\
\text { molecules }\end{array}$ & CxFy & $\begin{array}{l}\text { CHxFy, } \\
\text { CClxFy }\end{array}$ & NFx, SFy & SixYz & Total \\
\hline Species & 9 & 10 & 7 & 4 & 7 & 28 & 4 & 7 & 14 & 90 \\
\hline TCS & 2 & 6 & 4 & 4 & 7 & 5 & 2 & 3 & 4 & 37 \\
\hline ES & 2 & 5 & 4 & 2 & 1 & 6 & 2 & 2 & 2 & 26 \\
\hline MT & 2 & 4 & 3 & 2 & 1 & 5 & 2 & 2 & 2 & 23 \\
\hline DCS & 2 & 4 & 3 & 2 & 2 & 103 & 15 & 2 & 4 & 137 \\
\hline TICS & 9 & 7 & 7 & 4 & 4 & 8 & 4 & 3 & 10 & 56 \\
\hline PICS & 21 & 29 & 35 & 9 & 18 & 45 & 34 & 20 & 57 & 268 \\
\hline TDCS & 0 & 2 & 1 & 0 & 0 & 4 & 1 & 0 & 2 & 10 \\
\hline NDCS & 0 & 0 & 0 & 0 & 0 & 1 & 0 & 0 & 4 & 5 \\
\hline TACS & 0 & 4 & 2 & 1 & 0 & 4 & 1 & 0 & 0 & 12 \\
\hline DACS & 0 & 4 & 9 & 2 & 0 & 1 & 2 & 3 & 0 & 21 \\
\hline VI & 0 & 11 & 3 & 1 & 0 & 1 & 2 & 0 & 0 & 18 \\
\hline $\mathrm{RO}$ & 0 & 7 & 0 & 1 & 0 & 0 & 0 & 0 & 0 & 8 \\
\hline EX & 0 & 26 & 2 & 0 & 0 & 0 & 0 & 0 & 0 & 28 \\
\hline
\end{tabular}

TCS denotes the total cross section; ES, elastic scattering cross section; MT, momentum transfer cross section; DCS, differential cross section; TICS, total ion cross section; PICS, partial ion cross section; TDCS, total dissociation cross section; NDCS, neutral dissociation cross section; TACS, total attachment cross section; DACS, dissociative attachment cross section; VI, vibrational excitation cross section; RO, rotational excitation cross section; and EX, excitation cross section.

Table III. List of surface reaction data.

\begin{tabular}{|c|c|c|c|c|c|c|c|c|c|c|c|c|c|c|c|c|c|c|c|c|}
\hline $\begin{array}{l}\text { Sputtering } \\
\text { Yield }\end{array}$ & $\begin{array}{l}\text { Target } \\
\text { Count }\end{array}$ & $\mathrm{Be}$ & C & $\mathrm{Al}$ & $\mathrm{Si}$ & $\mathrm{Ti}$ & $\mathrm{V}$ & $\mathrm{Fe}$ & $\mathrm{Ni}$ & $\mathrm{Cu}$ & $\mathrm{Ge}$ & $\mathrm{Zr}$ & $\mathrm{Nb}$ & Mo & $\mathrm{Pd}$ & $\mathrm{Ag}$ & $\mathrm{Ta}$ & $\mathrm{W}$ & $\mathrm{Pt}$ & $\mathrm{Au}$ \\
\hline $\mathrm{Ar}$ & 15 & & 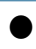 & 0 & 0 & 0 & 0 & 0 & 0 & & 0 & & 0 & 0 & 0 & 0 & 0 & 0 & 0 & \\
\hline $\mathrm{Kr}$ & 15 & & 0 & & 0 & 0 & 0 & 0 & 0 & & 0 & 0 & & 0 & 0 & 0 & 0 & 0 & 0 & 0 \\
\hline $\mathrm{Xe}$ & 15 & 0 & 0 & & 0 & 0 & 0 & 0 & 0 & & 0 & & & 0 & 0 & 0 & 0 & 0 & 0 & 0 \\
\hline
\end{tabular}

Table IV. List of thermodynamic data.

\begin{tabular}{|c|c|c|c|c|c|c|}
\hline Group & $\begin{array}{l}\text { Noble } \\
\text { gas }\end{array}$ & $\begin{array}{c}\mathrm{CxFy} \\
(\mathrm{x}=0-4 \\
\mathrm{y}=1-8)\end{array}$ & $\begin{array}{l}\mathrm{CxHyFz} \\
(\mathrm{x}=0-1 \\
\mathrm{y}=1-2 \\
\mathrm{z}=1-2)\end{array}$ & $\begin{array}{l}\mathrm{CxOyFz} \\
(x=0-1 \\
y=1-3 \\
z=0-2)\end{array}$ & $\begin{array}{c}\text { NFx } \\
(x=0-3)\end{array}$ & Total \\
\hline Chemical species & 1 & 52 & 9 & 8 & 3 & 73 \\
\hline Ion species & 2 & 86 & 15 & 10 & 3 & 116 \\
\hline EA & 1 & 43 & 9 & 3 & 2 & 59 \\
\hline IP & 1 & 52 & 9 & 3 & 3 & 68 \\
\hline$a$ & 1 & 76 & 14 & 3 & 3 & 97 \\
\hline $\mathrm{L}^{-} \mathrm{J}\left(\sigma, \varepsilon / \mathrm{k}_{\mathrm{B}}\right)$ & 1 & 13 & 7 & 4 & 0 & 25 \\
\hline $\begin{array}{l}\text { Thermodynamic } \\
\text { data }\end{array}$ & 2 & 28 & 15 & 10 & 0 & 55 \\
\hline
\end{tabular}

\subsection{Available function}

Figure 3 shows the results obtained after "Xe atom" was entered in the search box. Unlike the previous system, the search is integrated, and therefore, all data information is displayed on one page. When the process tab is clicked, the retrieved data is visualized graphically, as

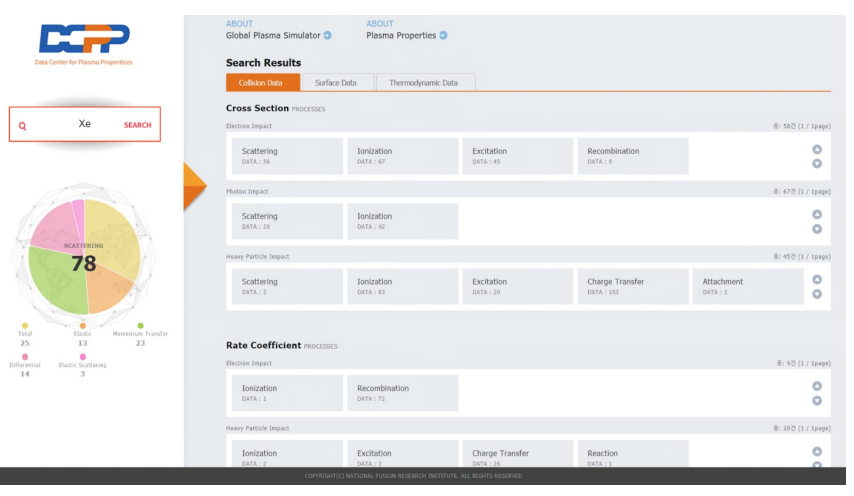

Figure 3. (Color online) Search result page of the new system.

shown in Fig. 4. Whether visualizing it is necessary can be determined by clicking on the property number at the top of the graph or by selecting a checkbox among the lists below the graph. Moreover, when the symbol on the graph is clicked, the raw data information is visualized. When a user wants to visualize only one reaction property among the retrieved data, the user can obtain the visualized data by clicking a graph icon among the lists below the graph, as shown in Fig. 


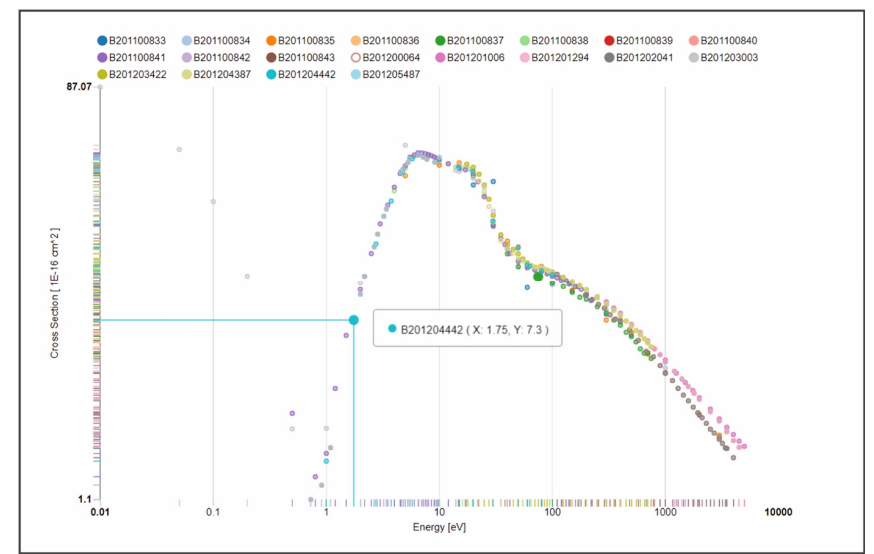

Figure 4. (Color online) Example of the visualization of retrieved data.

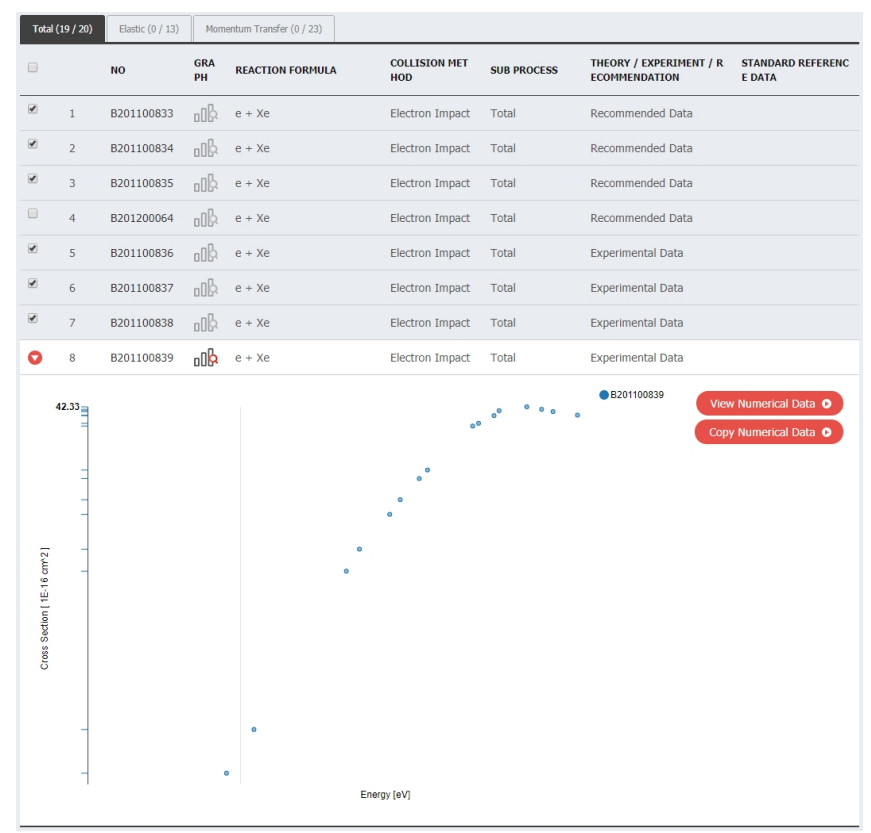

Figure 5. (Color online) Example of the visualization of a selected process.

\begin{tabular}{|c|c|c|c|c|}
\hline Molecular & $\begin{array}{l}\text { Rational } \\
\end{array}$ & temperature(K) & $200-1000$ & $1000-6000$ \\
\hline \multirow[t]{7}{*}{$c_{4}$} & $\mathrm{c}_{4}$ & at & 3.335-01 & $9,996+00$ \\
\hline & & az & 3.3000 .02 & $2.99 E-03$ \\
\hline & & as & -3.32205 & 1.020 .06 \\
\hline & & 94 & 2.14500 & L.81E: 10 \\
\hline & & as & $4,735 \mathrm{E}-12$ & $-1.09 E-14$ \\
\hline & & b1 & $5.692-03$ & 3.44t +03 \\
\hline & & b2 & $2.125+01$ & $2 \pi €+01$ \\
\hline & & $\begin{array}{c}\frac{C_{p}(T)}{R}=a_{1}+a \\
\frac{H^{0}(T)}{R(T)}=a_{1}+a_{2} \frac{T}{2}+ \\
R T \\
\frac{S^{0}(T)}{R}=a_{1} l h T+a_{2} T \\
C_{p}: \text { heat } \\
H^{0} \quad: \\
S^{0}: \\
T: \\
R: \quad \text { gas const }\end{array}$ & 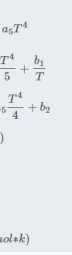 & \\
\hline
\end{tabular}

Figure 6. (Color online) Example of the thermodynamic data search.

5. Numerical data can be obtained by clicking the icon at the top right of the graph. Further, bibliographic data can be obtained by clicking the process number among the lists below the graph. On the same page, the user can download the data in the XSAMS format, which is an atomic and molecule data exchange format. Surface reaction data can be retrieved in the same way as collision cross sections or rate coefficients search.

Thermodynamic data can be used to calculate the radical temperature or molar fraction in the plasma chamber. In our study, thermodynamic data were obtained by quantum chemistry theory calculations as shown in Fig. 6. The system provides geometrical Cartesian coordinates of the atom or molecule, adiabatic electron affinity, vertical electron affinity, vertical electron detachment energy, vertical ionization potential, adiabatic ionization potential, polarizability, thermodynamic data (raw), and polynomial fitting parameters of the obtained thermodynamic raw data.

\section{Conclusions}

Plasma property data are used as basic data for developing simulators and plasma modelling, particularly in the semiconductor field. Most existing data such as atomic and molecular scattering cross section data of the plasma are based on reaction data in the gaseous state about plasma, and they are insufficient to meet the diverse requirements of the industry and the researchers. Further, surface reaction data are reaction data about neutral atoms, electrons, or ions that react with substances in the liquid or solid states. These data can be used to characterize the reaction mechanism of the plasma properties for applications in thin film deposition and sputtering. Further, thermodynamic data can be used to identify thermodynamic reactions between atoms and molecules and produce new simulation results that generate particular types of gas used to create the plasma. Therefore, the database was designed to manage data related to plasma material reaction including plasma impact and surface reaction data and plasma chemistry data such as thermodynamic data for the user.

In future works, data related to plasma optical signal analysis from diagnostic device will be added to the system so that it can be managed. Moreover, we plan to create an interface for data exchange and apply it to the system to participate in the VAMDC consortium.

\section{Acknowledgements}

This work was supported by the R\&D Program of "Plasma Big Data ICT Convergence Technology Research Project" through the National Fusion Research Institute of Korea (NFRI) funded by Government funds.

\section{References}

[1] A. Grill, Cold Plasma in Materials Fabrication from Fundamentals to Applications (John Willy \& Sons, Inc, 1994), pp. 1-27.

[2] IAEA Atomic and Molecular Data Unit https://www-amdis.iaea.org (accessed Jan. 31, 2020).

[3] IAEA ALADDIN http://www-amdis.iaea.org/ALADDIN (accessed Jan. 31, 2020).

[4] R. A. Hulse, AIP Conf. Proc. 206, 63 (1990).

[5] D. P. Humbert, A. L. Nichols, and O. Schwerer, Proceedings of the 14th International Conference on Radionuclide Metrology and its Applications (2004), Vol. 60, pp. 311-316.

[6] IAEA AMBDAS http://www-amdis.iaea.org/AMBDAS (accessed 
Jan. 31, 2020).

[7] Y. V. Ralchenkol, R. E. H. Clark, and J. A. Stephens, 5th Israeli Conference on Plasma Science and Applications Book of Abstracts (2002), pp. 37.

[8] IAEA GENIE http://www-amdis.iaea.org/GENIE (accessed Jan. 31, 2020).

[9] VAMDC http://www.vamdc.org/ (accessed Jan. 31, 2020).

[10] M. L. Dubernet, B. K. Antony, Y. A. Ba, Y. L. Babikov, K. Bartschat, V. Boudon, B. J. Braams, H. K. Chung, F. Daniel, F. Delahaye, and G. Del Zanna, J. Phys. B: At., Mol. Opt. Phys. 49, 1 (2016).

[11] G. Rixon, M. Dubernet, N. Piskunov, N. Walton, N. Mason, P. Sidaner, S. Schlemmer, J. Tennyson, A. Akram, K. Benson, and J. Bureau, AIP Conf. Proc. 1344, 107 (2011).

[12] Y. Ralchenko, R. E. H. Clark, M. L. Dubernet, S. Gagarin, D.
Humbert, P. A. Loboda, N. Moreau, E. Roueff, and D. R. Schultz, AIP Conf. Proc. 1125, 207 (2009).

[13] J. Tennyson, S. Rahimi, C. Hill, L. Tse, A. Vibhakar, D. AkelloEgwel, D. B. Brown, A. Dzarasova, J. R. Hamilton, D. Jaksch, and S. Mohr, Plasma Sources Sci. Technol. 26, 055014 (2017).

[14] L. C. Pitchford, L. L. Alves, K. Bartschat, S. F. Biagi, M. C. Bordage, I. Bray, C. E. Brion, M. J. Brunger, L. Campbell, A. Chachereau, and B. Chaudhury, Plasma Processes and Polymers 14, 1600098 (2017).

[15] Data Center for Plasma Properties http://dcpp.nfri.re.kr (accessed Jan. 31, 2020).

[16] National Center for Standard Reference Data http://www.srd.re.kr (accessed Jan. 31, 2020). 